CARPATHIAN J. MATH.

Volume 38 (2022), No. 2,

Pages 417 - 429

\title{
Classification of pure metallic metric geometries
}

\author{
FERnANDo EtAyo, ARACELI DEFrAncisco and RAFAEL SANTAMARía
}

\begin{abstract}
Metallic Riemannian manifolds with null trace and metallic Norden manifolds are generalizations of almost product Riemannian and almost golden Riemannian manifolds with null trace and almost Norden and almost Norden golden manifolds respectively. All these pure metrics geometries can be unified under the notion of $\alpha$-metallic metric manifold. We classify this kind of manifolds in a consistent way with the wellknown classifications of almost product Riemannian manifolds with null trace and almost Norden manifolds. We also characterize all classes of $\alpha$-metallic metric manifolds by means of the first canonical connection which is a distinguished adapted connection.
\end{abstract}

\section{INTRODUCTION}

The golden ratio is the most known number of the family of "real metallic numbers" which are the positive root of the polynomials $x^{2}-p x-q, p, q \in \mathbb{N}, p^{2}+4 q>0$; i.e., the numbers of the form $\frac{p+\sqrt{p^{2}+4 q}}{2}$. Analogously one can introduce the "complex metallic numbers" as the family of numbers of the form $\frac{p+i \sqrt{p^{2}+4 q}}{2}$, where $p, q \in \mathbb{N}, p^{2}+4 q>0$, which are one of the roots of the polynomials $x^{2}-p x+\frac{2 p^{2}+4 q}{4}$. Then the golden (resp. complex golden) ratio is the real (resp. complex) metallic number when $p=q=1$.

Both families of numbers can be unified as follows. According to $\alpha=-1$ or $\alpha=1$, the numbers of the form $\frac{p+\sqrt{\alpha\left(p^{2}+4 q\right)}}{2}$, where $p, q \in \mathbb{N}, p^{2}+4 q>0$, called " $\alpha$-metallic numbers", are the complex and real metallic numbers respectively, which are one of the roots of the polynomials

$$
x^{2}-p x-\frac{p^{2}(\alpha-1)+4 q \alpha}{4} p, q \in \mathbb{N}, p^{2}+4 q>0 .
$$

Almost complex and almost product structures on manifolds are the two most studied polynomial structures of degree 2. Inspired by them, Crasmareanu and Hreţcanu in [4] introduced almost complex golden and almost golden structures on manifolds which are also polynomial structures of degree 2 . The above structures can be unified under the notions of $\alpha$-structure and $\alpha$-golden structure where $\alpha \in\{-1,1\}$, which are polynomial structures of degree two whose characteristic polynomials are

$$
x^{2}-\alpha, x^{2}-x-\frac{5 \alpha-1}{4},
$$

respectively. Note that an $\alpha$-structure is a tensor field $J$ of type $(1,1)$ satisfying $J^{2}=\alpha I d$, where $I d$ is the identity tensor field of type $(1,1)$; i.e., if $\alpha=-1$ (resp. $\alpha=1$ ) then $J$ is an almost complex (resp. almost product) structure. In the case of an $\alpha$-golden structure, an almost complex golden structure is a tensor field $\varphi$ of type $(1,1)$ satisfying $\varphi^{2}=\varphi-\frac{3}{2} I d$

Received: 06.05.2021. In revised form: 01.12.2021. Accepted: 08.12.2021

1991 Mathematics Subject Classification. 53C15, 53C07, 53C05.

Key words and phrases. $\alpha$-metallic structure, $\alpha$-metallic metric manifold, first canonical connection, classification.

Corresponding author: Fernando Etayo; fernando.etayo@unican.es 
if $\alpha=-1$, while if $\alpha=1$ a tensor field $\varphi$ of type $(1,1)$ satisfying $\varphi^{2}=\varphi+I d$ is an almost golden structure.

The generalization of golden and complex golden ratio give rise to the introduction of $\alpha$-metallic numbers. Analogously, the notion of $\alpha$-metallic structure on manifolds can be viewed as the generalization of $\alpha$-structures and $\alpha$-golden structures. Given $p, q \in \mathbb{N}$, $p^{2}+4 q>0$, a polynomial structure $\varphi$ of degree 2 on $M$ whose characteristic polynomial is (1.1); i.e., if it satisfies

$$
\varphi^{2}=p \varphi+\frac{p^{2}(\alpha-1)+4 q \alpha}{4} I d
$$

is called an $\alpha$-metallic structure. In this case $(M, \varphi)$ is called an $\alpha$-metallic manifold. Note that if $\alpha=-1$, almost complex and almost complex golden structures are $\alpha$-metallic structures when $(p, q)=(0,1)$ and $(p, q)=(1,1)$ respectively. In the case $\alpha=1$, almost product and almost golden structures are $\alpha$-metallic structures when $(p, q)=(0,1)$ and $(p, q)=(1,1)$ respectively. Complex and real metallic structures according $\alpha=-1$ and $\alpha=1$ were introduced in [2] and [11] respectively.

Now, we show the closely relationship between $\alpha$-structures and $\alpha$-metallic structures on a manifold. Given $p, q \in \mathbb{N}, p^{2}+4 q>0$, if $J$ is an $\alpha$-structure on $M$ then $\varphi_{J}$ is an $\alpha$-metallic structure, and reciprocally, if $\varphi$ is an $\alpha$-metallic structure on $M$ then $J_{\varphi}$ is an $\alpha$-structure, where

$$
\varphi_{J}=\frac{p}{2} I d+\frac{\alpha \sqrt{p^{2}+4 q}}{2} J, \quad J_{\varphi}=\frac{(-\alpha)}{\sqrt{p^{2}+4 q}}(p I d-2 \varphi)
$$

(see [2, Sec. 5] and [11, Prop. 3.2]). A direct calculus shows that $\varphi_{J_{\varphi}}=\varphi$ and $J_{\varphi_{J}}=J$, thus, there exist a 1:1 correspondence between $\alpha$-structures and $\alpha$-metallic structures as follows:

$$
J \mapsto \varphi_{J}, \quad \varphi \mapsto J_{\varphi}
$$

The next step is to introduce pure metrics on $\alpha$-metallic manifolds. Let $(M, \varphi)$ be an $\alpha$-metallic manifold and let $g$ be a pseudo-Riemannian metric on $M$ if $\alpha=-1$ or a Riemannian metric if $\alpha=1$. One says that $g$ is a pure metric if it satisfies one of the two equivalent conditions

$$
g(\varphi X, Y)=g(X, \varphi Y), g(\varphi X, \varphi Y)=p g(\varphi X, Y)+\frac{p^{2}(\alpha-1)+4 q \alpha}{4} g(X, Y),
$$

for all vector fields $X, Y$ on $M$. In the above conditions $(M, \varphi, g)$ is an almost (pseudo)Riemannian metallic manifold (see [2] and [11]).

It is easy to prove that the following conditions are equivalent

$$
g(\varphi X, Y)=g(X, \varphi Y), g\left(J_{\varphi} X, Y\right)=g\left(X, J_{\varphi} Y\right), \quad \forall X, Y \in \mathfrak{X}(M) .
$$

These identities allow to claim that the metric $g$ is pure respect to $\varphi$ and $J_{\varphi}$ simultaneously.

In the above conditions, if $\alpha=-1$ then $g$ is a pseudo-Riemannian metric of signature $(n, n)$ and $\left(M, J_{\varphi}, g\right)$ is an almost Norden manifold, while if $\alpha=1$ and the trace of the polynomial structure of the almost product Riemannian manifold $\left(M, J_{\varphi}, g\right)$ vanishes then the manifold has also even dimension. This last kind of metric manifolds are called almost product Riemannian manifolds with null trace.

On the other hand, there are some articles devoted to study the case characterized by

$$
g(\varphi X, Y)=-g(X, \varphi Y), \forall X, Y \in \mathfrak{X}(M),
$$

i.e., metallic manifolds endowed with anti-pure metrics (see [1] and [13]). 
Almost Norden manifolds and almost product Riemannian manifolds with null trace can be unified under the notion of $\alpha$-metric manifolds as follows. Given an even dimensional manifold $M$ endowed with an $\alpha$-structure $J$ and a pseudo-Riemannian metric $g$ (in fact, a Riemannian metric if $\alpha=1$ such that $J$ has null trace), one says that $(M, J, g)$ is an $\alpha$-metric manifold if it satisfies one of the two following equivalent conditions

$$
g(J X, J Y)=\alpha g(X, Y), g(J X, Y)=g(X, J Y), \quad \forall X, Y \in \mathfrak{X}(M) .
$$

Note that $\alpha$-metric manifolds are $(\alpha, \varepsilon)$-metric manifolds such that $\alpha \varepsilon=1$ (see [7]). The introduction of the notion of $\alpha$-metric manifold allows us to classify in a unified way almost Norden manifolds and almost product Riemannian manifolds with null trace.

Hereinafter, we restrict our study to even dimensional almost (pseudo)-Riemannian metallic manifolds $(M, \varphi, g)$ such that $\left(M, J_{\varphi}, g\right)$ be an $\alpha$-metric manifold. We call $\alpha$ metallic metric manifolds to this kind of manifolds. In the case $\alpha=-1$ we say that $(M, \varphi, g)$ is an almost metallic Norden manifold, while if $\alpha=1$ we say that $(M, \varphi, g)$ is an almost metallic Riemannian manifold with null trace.

The bijection (1.4) between $\alpha$-structures and $\alpha$-metallic structures can be extended to the metric case because of (1.5). Given $p, q \in \mathbb{N}, p^{2}+4 q>0$, the $\alpha$-metallic metric manifold $(M, \varphi, g)$ and the $\alpha$-metric manifold $\left(M, J_{\varphi}, g\right)$ are $1: 1$ related in the same way as the $\varphi$ and $J_{\varphi}$.

This bijection allows to classify $\alpha$-metallic metric manifolds starting from the classification of $\alpha$-metric manifolds as follows: an $\alpha$-metallic metric manifold $(M, \varphi, g)$ belongs to certain class of manifolds of those showed in Theorem 2.1 if and only if the $\alpha$-metric manifold $\left(M, J_{\varphi}, g\right)$ belongs to this class of manifolds. To classify $\alpha$-metallic metallic manifolds is the main goal of our paper. This one should be consistent with the classifications of almost Norden manifolds and almost product Riemannian manifolds with null trace obtained in [9] and [12], because an $\alpha$-metallic metric manifold such that $(\alpha, p, q)=(-1,0,1)$ is an almost Norden manifold, while if $(\alpha, p, q)=(1,0,1)$ the $\alpha$-metric metallic manifold is an almost product Riemannian manifold with null trace.

The Levi-Civita connection plays a key role in the classification of $\alpha$-metric manifolds. But there exist another connections which allow characterize easily some classes of this kind of pure metric manifolds. One of them is the first canonical connection of an $\alpha$ metric manifold $(M, J, g)$ which is an adapted connection to $(J, g)$; i.e., it is a connection that parallelize the $\alpha$-structure $J$ and the metric $g$ (see [7]). The first canonical connection $\nabla^{0}$ of $(M, J, g)$ and its torsion tensor $\mathrm{T}^{0}$ are given by

$$
\begin{aligned}
\nabla_{X}^{0} Y & =\nabla_{X}^{\mathrm{g}} Y+\frac{(-\alpha)}{2}\left(\nabla_{X}^{\mathrm{g}} J\right) J Y, \\
\mathrm{~T}^{0}(X, Y) & =\frac{(-\alpha)}{2}\left(\left(\nabla_{X}^{\mathrm{g}} J\right) J Y-\left(\nabla_{Y}^{\mathrm{g}} J\right) J X\right), \quad \forall X, Y \in \mathfrak{X}(M),
\end{aligned}
$$

where $\nabla^{\mathrm{g}}$ denotes the Levi-Civita connection of the metric $g$.

It is well-known that the Nijenhuis tensor $N_{J}$ of an almost Norden or an almost product Riemannian manifold $(M, J, g)$ can be expressed as follows:

$$
\begin{aligned}
N_{J}(X, Y) & =J^{2}[X, Y]+[J X, J Y]-J[J X, Y]-J[X, J Y] \\
& =\left(\nabla_{X}^{\mathrm{g}} J\right) J Y+\left(\nabla_{J X}^{\mathrm{g}} J\right) Y-\left(\nabla_{Y}^{\mathrm{g}} J\right) J X-\left(\nabla_{J Y}^{\mathrm{g}} J\right) X, \quad \forall X, Y \in \mathfrak{X}(M) .
\end{aligned}
$$

Starting from the above identities it is easy to prove the next one

$$
\mathrm{T}^{0}(J X, J Y)+\alpha \mathrm{T}^{0}(X, Y)=-\frac{1}{2} N_{J}(X, Y), \forall X, Y \in \mathfrak{X}(M),
$$


(see also [7, Prop. 5.1]), thus, an $\alpha$-metric manifold $(M, J, g)$ is integrable if and only if the torsion tensor of the first canonical connection satisfies $\mathrm{T}^{0}(J X, J Y)+\alpha \mathrm{T}^{0}(X, Y)=0$, for all vector fields $X, Y$ on $M$.

In this paper we also characterize all classes of $\alpha$-metric manifolds using the first canonical connection instead of the Levi-Civita connection.

The above 1: 1 correspondence is a very useful tool. Mathematical objects univocally attached to an $\alpha$-metric structure can be associated to its corresponding $\alpha$-metric metallic structure. For instance, this property allows to introduce the first canonical connection of $(M, \varphi, g)$ as the first canonical connection of $\left(M, J_{\varphi}, g\right)$. We also characterize all classes of $\alpha$-metric metallic manifolds using this connection.

The organization of the paper is as follows:

In Section 2 we will classify $\alpha$-metric manifolds (Theorem 2.1). Later, the torsion tensor of the first canonical will allow us to get new defining conditions of all classes of $\alpha$-metric manifolds (Theorem 2.2).

In Section 3 we will focus on $\alpha$-metallic metric manifolds. Given an $\alpha$-metallic metric manifold $(M, \varphi, g)$, we will show useful relationships between tensors and forms defined from the $\alpha$-metallic metric structure $(\varphi, g)$ with other ones defined from its corresponding $\alpha$-metric structure $\left(J_{\varphi}, g\right)$, which allow us to classify the $\alpha$-metallic metric manifolds according to Definition 3.1 (Theorem 3.3). Later, in Definition 3.4, we will introduce the first canonical of an $\alpha$-metallic metric manifold, which allows us to characterize all classes of $\alpha$-metallic metric manifolds using its torsion tensor (Theorem 3.4).

We will consider smooth manifolds and operators being of class $C^{\infty}$. As in this Introduction, $\mathfrak{X}(M)$ denotes the module of vector fields of a manifold $M$.

\section{CLASSIFICATION OF $\alpha$-METRIC MANIFOLDS}

We will show the classification of $\alpha$-metric manifolds that unifies the previous classifications of almost Norden and almost product Riemannian manifolds with null trace obtained by Ganchev and Borisov and Staikova and Gribachev in [9] and [12] respectively. All classes of $2 n$-dimensional $\alpha$-metric manifolds are characterized by imposing conditions that involve tensors and forms introduced defined from $\nabla^{\mathrm{g}} J$.

The tensor field $\nabla^{\mathrm{g}} J$ of type $(1,2)$ is defined as follows:

$$
\left(\nabla_{X}^{\mathrm{g}} J\right) Y=\nabla_{X}^{\mathrm{g}} J Y-J \nabla_{X}^{\mathrm{g}} Y, \forall X, Y \in \mathfrak{X}(M) .
$$

As direct consequence of the above identity it is easy to prove the next result.

Lemma 2.1. Let $(M, J, g)$ be an $\alpha$-metric manifold. The following relations hold:

$$
\begin{aligned}
g\left(\left(\nabla_{X}^{\mathrm{g}} J\right) Y, Z\right) & =g\left(\left(\nabla_{X}^{\mathrm{g}} J\right) Z, Y\right), \\
g\left(\left(\nabla_{X}^{\mathrm{g}} J\right) J Y, Z\right) & =-g\left(\left(\nabla_{X}^{\mathrm{g}} J\right) Y, J Z\right), \\
g\left(\left(\nabla_{X}^{\mathrm{g}} J\right) J Y, Z\right) & =-g\left(\left(\nabla_{X}^{\mathrm{g}} J\right) J Z, Y\right), \quad \forall X, Y, Z \in \mathfrak{X}(M) .
\end{aligned}
$$

The tensor field $\Phi$ of type $(0,2)$ and its covariant derivative respect to the Levi-Civita connection of $g$ are given by

$$
\begin{aligned}
\Phi(X, Y) & =g(J X, Y), \\
\left(\nabla_{X}^{\mathrm{g}} \Phi\right)(Y, Z) & =g\left(\left(\nabla_{X}^{\mathrm{g}} J\right) Y, Z\right), \forall X, Y, Z \in \mathfrak{X}(M) .
\end{aligned}
$$

As direct consequence of (2.13) the above tensor field of type $(0,3)$ satisfies

$$
\left(\nabla_{X}^{\mathrm{g}} \Phi\right)(J Y, Y)=g\left(\left(\nabla_{X}^{\mathrm{g}} J\right) J Y, Y\right)=0, \quad \forall X, Y \in \mathfrak{X}(M) .
$$



lows:

$$
\delta \Phi(X)=\sum_{i, j=1}^{2 n} g^{i j} g\left(\left(\nabla_{X_{i}}^{\mathrm{g}} J\right) X_{j}, X\right), \quad \forall X \in \mathfrak{X}(M),
$$

where $\left(X_{1}, \ldots, X_{2 n}\right)$ is a local basis of $T M$ and the matrix $\left(g^{i j}\right)_{1 \leq i, j \leq 2 n}$ is the inverse matrix of $\left(g\left(X_{i}, X_{j}\right)\right)_{1 \leq i, j \leq 2 n}$.

The vanishing of the tensor field $\nabla^{\mathrm{g}} \Phi$ and the 1 -form $\delta \Phi$ characterize the classes $\mathcal{W}_{0}$ and $\mathcal{W}_{2} \oplus \mathcal{W}_{3}$ of almost Norden manifolds and almost product Riemannian manifolds with null trace. Integrable and quasi-Kähler manifolds are characterized by the vanishing of the Nijenhuis tensor of $J$ and the tensor field $\widetilde{N}_{J}$, which can be expressed as follows:

$$
\widetilde{N}_{J}(X, Y)=\left(\nabla_{X}^{\mathrm{g}} J\right) J Y+\left(\nabla_{J X}^{\mathrm{g}} J\right) Y+\left(\nabla_{Y}^{\mathrm{g}} J\right) J X+\left(\nabla_{J Y}^{\mathrm{g}} J\right) X, \quad \forall X, Y \in \mathfrak{X}(M) .
$$

Here we will obtain new characteristic conditions of the classes $\mathcal{W}_{3}$ and $\mathcal{W}_{1} \oplus \mathcal{W}_{3}$ different from the original ones showed in [9] and [12], which are identities (2.18) and (2.21).

Lemma 2.2. Let $(M, J, g)$ be an $\alpha$-metric manifold. The following conditions are equivalent:

$$
\begin{aligned}
\widetilde{N}_{J}(X, Y) & =0, \quad \forall X, Y \in \mathfrak{X}(M), \\
\left(\nabla_{X}^{\mathrm{g}} J\right) J X+\left(\nabla_{J X}^{\mathrm{g}} J\right) X & =0, \quad \forall X \in \mathfrak{X}(M) .
\end{aligned}
$$

Proof. If the tensor $\tilde{N}_{J}$ vanishes, bearing in mind (2.17) it is obvious that identity (2.19) is true.

A direct calculus shows that the tensor field $\widetilde{N}_{J}$ satisfies the following identity

$$
\begin{aligned}
\tilde{N}_{J}(X, Y) & =\left(\nabla_{X+Y}^{\mathrm{g}} J\right)(J(X+Y))+\left(\nabla_{J(X+Y)}^{\mathrm{g}} J\right)(X+Y) \\
& -\left(\left(\nabla_{X}^{\mathrm{g}} J\right) J X+\left(\nabla_{J X}^{\mathrm{g}} J\right) X+\left(\nabla_{Y}^{\mathrm{g}} J\right) J Y+\left(\nabla_{J Y}^{\mathrm{g}} J\right) Y\right), \quad \forall X, Y \in \mathfrak{X}(M) .
\end{aligned}
$$

Thus, if $\nabla^{\mathrm{g}} J$ satisfies (2.19), bearing in mind (2.20), one gets that the tensor field $\widetilde{N}_{J}$ vanishes.

Lemma 2.3. Let $(M, J, g)$ be a $2 n$-dimensional $\alpha$-metric manifold. The following conditions are equivalent:

$$
\begin{aligned}
& \mathfrak{S}\left(\nabla_{X}^{\mathrm{g}} \Phi\right)(Y, Z) \\
&=\frac{1}{n X Y Z} \underset{\mathfrak{S}_{Y Z}}{ }(g(X, Y) \delta \Phi(Z)-\alpha g(X, J Y) \delta \Phi(J Z)), \\
& g\left(\left(\nabla_{X}^{\mathrm{g}} J\right) J X, Y\right)+g\left(\left(\nabla_{J X}^{\mathrm{g}} J\right) X, Y\right)=\frac{1}{n}(g(J X, X) \delta \Phi(Y)-g(X, X) \delta \Phi(J Y)),
\end{aligned}
$$

for all $X, Y, Z$ vector fields on $M$, where $\underset{X Y Z}{\mathfrak{S}}$ denotes the cyclic sum by $X, Y, Z$.

Proof. As direct consequence of (1.6), (2.11), (2.14) and (2.15), if one evaluates identity (2.21) on $(X, Y, J X)$ then one obtains (2.22).

Given $X, Y, Z$ vector fields on $M$, taking into account (2.11), (2.12) and (2.17), one has

$$
\begin{aligned}
g\left(\tilde{N}_{J}(X, Y), J Z\right) & =-\alpha g\left(\left(\nabla_{X}^{\mathrm{g}} J\right) Y, Z\right)-g\left(\left(\nabla_{J X}^{\mathrm{g}} J\right) J Y, Z\right) \\
& \left.-\alpha g\left(\left(\nabla_{Y}^{\mathrm{g}} J\right) Z, X\right)\right)+g\left(\left(\nabla_{J Y}^{\mathrm{g}} J\right) J Z, X\right),
\end{aligned}
$$

then one obtains

$$
\underset{X Y Z}{\mathfrak{S}} g\left(\widetilde{N}_{J}(X, Y), J Z\right)=-2 \alpha \underset{X Y Z}{\mathfrak{S}} g\left(\left(\nabla_{X}^{\mathrm{g}} J\right) Y, Z\right)
$$


Given $X, Y, Z$ vector fields on $M$, identities (1.6), (2.20) and (2.22) lead to the next ones

$$
\begin{aligned}
g\left(\tilde{N}_{J}(X, Y), Z\right) & =\frac{1}{n}(g(J X+J Y, X+Y) \delta \Phi(Z)-g(X+Y, X+Y) \delta \Phi(J Z)) \\
& -\frac{1}{n}(g(J X, X) \delta \Phi(Z)-g(X, X) \delta \Phi(J Z)) \\
& -\frac{1}{n}(g(J Y, Y) \delta \Phi(Z)-g(Y, Y) \delta \Phi(J Z)) \\
& =\frac{2}{n}(g(J X, Y) \delta \Phi(Z)-g(X, Y) \delta \Phi(J Z)), \\
\underset{S}{S} g\left(\widetilde{N}_{J}(X, Y), J Z\right) & =\frac{2}{n} \underset{S Y Z}{\mathfrak{S}}(g(J X, Y) \delta \Phi(J Z)-\alpha g(X, Y) \delta \Phi(Z)),
\end{aligned}
$$

then, taking into account (1.6), (2.14) and (2.23), one obtains easily identity (2.21) from the above one.

The above results and the classifications of almost Norden manifolds and almost product Riemannian manifolds with null trace allow to classify both kind of metric manifolds in a unified way as follows.

Theorem 2.1. Let $(M, J, g)$ be a $2 n$-dimensional $\alpha$-metric manifold. Then one has the following classes of this kind of manifolds:

i) The class $\mathcal{W}_{0}$ or Kähler manifolds characterized by one of the following equivalent conditions

$$
\nabla^{\mathrm{g}} \Phi=0, \quad \nabla^{\mathrm{g}} J=0 .
$$

ii) The class $\mathcal{W}_{1}$ characterized by the condition

$$
\begin{aligned}
\left(\nabla_{X}^{\mathrm{g}} \Phi\right)(Y, Z) & =\frac{1}{2 n}(g(X, Y) \delta \Phi(Z)+g(X, Z) \delta \Phi(Y)) \\
& +\frac{(-\alpha)}{2 n}(g(X, J Y) \delta \Phi(J Z)+g(X, J Z) \delta \Phi(J Y)), \quad \forall X, Y, Z \in \mathfrak{X}(M) .
\end{aligned}
$$

iii) The class $\mathcal{W}_{2}$ characterized by the conditions

$$
\delta \Phi=0, N_{J}=0 .
$$

iv) The class $\mathcal{W}_{3}$ or quasi-Kähler manifolds characterized by the condition

$$
\left(\nabla_{X}^{\mathrm{g}} J\right) J X+\left(\nabla_{J X}^{\mathrm{g}} J\right) X=0, \forall X \in \mathfrak{X}(M) .
$$

v) The class $\mathcal{W}_{1} \oplus \mathcal{W}_{2}$ or integrable manifolds characterized by the condition

$$
N_{J}=0 \text {. }
$$

vi) The class $\mathcal{W}_{2} \oplus \mathcal{W}_{3}$ characterized by the condition

$$
\delta \Phi=0 .
$$

vii) The class $\mathcal{W}_{1} \oplus \mathcal{W}_{3}$ characterized by the condition

$$
g\left(\left(\nabla_{X}^{\mathrm{g}} J\right) J X, Y\right)+g\left(\left(\nabla_{J X}^{\mathrm{g}} J\right) X, Y\right)=\frac{1}{n}(g(X, J X) \delta \Phi(Y)-g(X, X) \delta \Phi(J Y)),
$$

for all vector fields $X, Y$ on $M$.

viii) The class $\mathcal{W}$ or the whole class of $\alpha$-metric manifolds.

To characterize all classes of $2 n$-dimensional $\alpha$-metric manifolds showed above by means of the first canonical connection, we need to extend the definition of the torsion form of the first canonical connection of almost product Riemannian manifold with null trace to the Norden case (see [8, Lemma 3.1]). 
Analogously to the 1 -form $\delta \Phi$ (see identity (2.16)), the torsion form $t^{0}$ of the first canonical connection of an $\alpha$-metric manifold $(M, J, g)$ can be locally defined as follows:

$$
t^{0}(X)=\sum_{i, j=1}^{2 n} g^{i j} g\left(\mathrm{~T}^{0}\left(X, X_{i}\right), X_{j}\right), \quad \forall X \in \mathfrak{X}(M),
$$

where $\left(X_{1}, \ldots, X_{2 n}\right)$ is a local basis of $T M$ and the matrix $\left(g^{i j}\right)_{1 \leq i, j \leq 2 n}$ is the inverse matrix of $\left(g\left(X_{i}, X_{j}\right)\right)_{1 \leq i, j \leq 2 n}$.

The next result shows the closely relationship between the 1-forms $\delta \Phi$ and $t^{0}$ on an $\alpha$-metric manifold.

Lemma 2.4. Let $(M, J, g)$ be a $2 n$-dimensional $\alpha$-metric manifold. Then one has

$$
\delta \Phi(X)=2 t^{0}(J X), \forall X \in \mathfrak{X}(M) .
$$

Proof. Given a vector field $X$ on $M$ and given a local basis $\left(X_{1}, \ldots, X_{2 n}\right)$, as $\left(g^{i j}\right)_{1 \leq i, j \leq 2 n}$ is a symmetric matrix, because of (2.13) one obtains

$$
\sum_{i, j=1}^{2 n} g^{i j} g\left(\left(\nabla_{J X}^{\mathrm{g}} J\right) J X_{i}, X_{j}\right)=0,
$$

thus, taking into account (1.8), (2.11) and (2.16), one obtains

$$
\begin{aligned}
g\left(\mathrm{~T}^{0}\left(J X, X_{i}\right), X_{j}\right) & =\frac{(-\alpha)}{2}\left(g\left(\left(\nabla_{J X}^{\mathrm{g}} J\right) J X_{i}, X_{j}\right)-\alpha g\left(\left(\nabla_{X_{i}}^{\mathrm{g}} J\right) X_{j}, X\right)\right), i, j=1, \ldots, 2 n, \\
t^{0}(J X) & =\frac{(-\alpha)}{2} \sum_{i, j=1}^{2 n} g^{i j} g\left(\left(\nabla_{J X}^{\mathrm{g}} J\right) J X_{i}, X_{j}\right)+\frac{1}{2} \sum_{i, j=1}^{2 n} g^{i j} g\left(\left(\nabla_{X_{i}}^{\mathrm{g}} J\right) X_{j}, X\right) \\
& =\frac{1}{2} \delta \Phi(X) .
\end{aligned}
$$

The next result provides defining conditions of all classes of $\alpha$-metric manifolds using the first canonical connection instead of the Levi-Civita connection like in Theorem 2.1.

Theorem 2.2. Let $(M, J, g)$ be a $2 n$-dimensional $\alpha$-metric manifold. The classes given in Theorem 2.1 can be characterized by means of the first canonical connection as follows:

i) The class $\mathcal{W}_{0}$ or Kähler manifolds characterized by the condition

$$
\mathrm{T}^{0}(X, Y)=0, \forall X, Y \in \mathfrak{X}(M) .
$$

ii) The class $\mathcal{W}_{1}$ characterized by the condition

$$
\mathrm{T}^{0}(X, Y)=\frac{1}{2 n}\left(t^{0}(X) Y-t^{0}(Y) X-\alpha t^{0}(J X) J Y+\alpha t^{0}(J Y) J X\right), \quad \forall X, Y \in \mathfrak{X}(M) .
$$

iii) The class $\mathcal{W}_{2}$ characterized by the conditions

$$
t^{0}(X)=0, \mathrm{~T}^{0}(J X, J Y)+\alpha \mathrm{T}^{0}(X, Y)=0, \forall X, Y \in \mathfrak{X}(M) .
$$

$i v)$ The class $\mathcal{W}_{3}$ or quasi-Kähler manifolds characterized by the condition

$$
g\left(\mathrm{~T}^{0}(J X, Y), J X\right)+\alpha g\left(\mathrm{~T}^{0}(X, Y), X\right)=0, \forall X, Y \in \mathfrak{X}(M) .
$$

v) The class $\mathcal{W}_{1} \oplus \mathcal{W}_{2}$ or integrable manifolds characterized by the condition

$$
\mathrm{T}^{0}(J X, J Y)+\alpha \mathrm{T}^{0}(X, Y)=0, \quad X, Y \in \mathfrak{X}(M) .
$$

vi) The class $\mathcal{W}_{2} \oplus \mathcal{W}_{3}$ characterized by the condition

$$
t^{0}(X)=0, \quad \forall X \in \mathfrak{X}(M) .
$$


vii) The class $\mathcal{W}_{1} \oplus \mathcal{W}_{3}$ characterized by the condition

$$
g\left(\mathrm{~T}^{0}(J X, Y), J X\right)+\alpha g\left(\mathrm{~T}^{0}(X, Y), X\right)=\frac{1}{n}\left(g(X, J X) t^{0}(J Y)-\alpha g(X, X) t^{0}(Y)\right),
$$

for all vector fields $X, Y$ on $M$.

viii) The class $\mathcal{W}$ or the whole class of $\alpha$-metric manifolds.

Proof.

i) The manifold $(M, J, g)$ belongs to the class $\mathcal{W}_{0}$ if and only if $\nabla^{\mathrm{g}} J=0$; i.e., $\nabla^{0}=\nabla^{\mathrm{g}}$. Therefore, $(M, J, g)$ is a Kähler manifold if and only if $\nabla^{0}$ is a torsion-free connection.

ii) Given $X, Y, Z$ vector fields on $M$, taking into account (1.6), (1.8), (2.14) and the defining condition (2.24) of the class $\mathcal{W}_{1}$, one gets

$$
\begin{aligned}
\frac{1}{2} g\left(\left(\nabla_{X}^{\mathrm{g}} J\right) J Y, Z\right) & =\frac{1}{4 n}(g(X, J Y) \delta \Phi(Z)+g(X, Z) \delta \Phi(J Y)), \\
& -\frac{1}{4 n}(g(X, Y) \delta \Phi(J Z)+g(X, J Z) \delta \Phi(Y)), \\
\frac{1}{2} g\left(\left(\nabla_{Y}^{\mathrm{g}} J\right) J X, Z\right) & =\frac{1}{4 n}(g(Y, J X) \delta \Phi(Z)+g(Y, Z) \delta \Phi(J X)), \\
& -\frac{1}{4 n}(g(Y, X) \delta \Phi(J Z)+g(Y, J Z) \delta \Phi(X)), \\
g\left(\mathrm{~T}^{0}(X, Y), Z\right) & =\frac{(-\alpha)}{4 n} g(\delta \Phi(J Y) X-\delta \Phi(J X) Y-\delta \Phi(Y) J X+\delta \Phi(X) J Y, Z),
\end{aligned}
$$

then, bearing in mind (2.25), one obtains the next identity which is an equivalent condition to $(2.26)$

$$
g\left(\mathrm{~T}^{0}(X, Y), Z\right)=\frac{1}{2 n} g\left(t^{0}(X) Y-t^{0}(Y) X-\alpha t^{0}(J X) J Y+\alpha t^{0}(J Y) J X, Z\right) .
$$

Reciprocally, given $X, Y, Z$ vector fields on $M$, as the first canonical connection is an adapted connection, according to [7, Prop. 3.6], one concludes the following equality

$$
g\left(\left(\nabla_{X}^{\mathrm{g}} J\right) Y, Z\right)=-g\left(\mathrm{~T}^{0}(X, J Y), Z\right)+g\left(\mathrm{~T}^{0}(J Y, Z), X\right)-g\left(\mathrm{~T}^{0}(Z, X), J Y\right),
$$

then, if one considers (1.6), (2.25), (2.29) and the above equality, by straightforward calculations one gets

$$
\begin{aligned}
g\left(\left(\nabla_{X}^{\mathrm{g}} J\right) Y, Z\right) & \left.=\frac{1}{n}\left(g(X, Y) t^{0}(J Z)+g(X, Z) t^{0}(J Y)\right)-g(X, J Y) t^{0}(Z)-g(X, J Z) t^{0}(Y)\right) \\
& =\frac{1}{2 n}(g(X, Y) \delta \Phi(Z)+g(X, Z) \delta \Phi(Y)) \\
& +\frac{(-\alpha)}{2 n}(g(X, J Y) \delta \Phi(J Z)+g(X, J Z) \delta \Phi(J Y))
\end{aligned}
$$

which is the defining condition (2.24) of the class $\mathcal{W}_{1}$.

iii) The characterization of the class $\mathcal{W}_{2}$ is a direct consequence of (1.10) and (2.25).

$i v$ ) Given $X, Y$ vector fields on $M$, identities (1.8), (2.13) and (2.15) carry to the following ones

$$
\begin{aligned}
g\left(\mathrm{~T}^{0}(X, Y), X\right) & =\frac{\alpha}{2} g\left(\left(\nabla_{X}^{\mathrm{g}} J\right) J X, Y\right), \\
g\left(\mathrm{~T}^{0}(J X, Y), J X\right) & =\frac{1}{2} g\left(\left(\nabla_{J X}^{\mathrm{g}} J\right) X, Y\right), \\
g\left(\mathrm{~T}^{0}(J X, Y), J X\right)+\alpha g\left(\mathrm{~T}^{0}(X, Y), X\right) & =\frac{1}{2} g\left(\left(\nabla_{X}^{\mathrm{g}} J\right) J X+\left(\nabla_{J X}^{\mathrm{g}} J\right) X, Y\right) .
\end{aligned}
$$

Thus, the defining condition (2.27) is a direct consequence of identity (2.19) and the above one. 
$v$ ) The defining condition (2.28) is a direct consequence of (1.10).

vi) The characterization of the class $\mathcal{W}_{2} \oplus \mathcal{W}_{3}$ is a direct consequence of (2.25).

vii) Given $X, Y$ vector fields on $M$, bearing in mind (2.22), (2.25) and(2.30), one obtains

$$
g\left(\mathrm{~T}^{0}(J X, Y), J X\right)+\alpha g\left(\mathrm{~T}^{0}(X, Y), X\right)=\frac{1}{n}\left(g(X, J X) t^{0}(J Y)-\alpha g(X, X) t^{0}(Y)\right),
$$

which is another characterization of the class $\mathcal{W}_{1} \oplus \mathcal{W}_{3}$.

The above theorem allows to recover the classification of almost Riemannian manifolds using the first canonical connection obtained in [8, Theor. 3.5] in the case $\alpha=1$.

\section{ClassificAtion OF $\alpha$-METALlic METRIC MANIFOLDS}

We want to classify the $\alpha$-metallic metric manifolds using the classification of $\alpha$-metric manifolds showed in Theorem 2.1 and the 1: 1 correspondence between $\alpha$-metric and $\alpha$-metallic metric manifolds.

Definition 3.1. Let $(M, \varphi, g)$ be an $\alpha$-metallic metric manifold. We say that $(M, \varphi, g)$ belongs to certain class according to the classification given in Theorem 2.1 if its corresponding $\alpha$-metric manifold $\left(M, J_{\varphi}, g\right)$ belongs to this class of manifolds.

Almost Norden golden and almost golden Riemannian manifolds with null trace were classified in [6] and [8] as it is indicated in the previous definition. There are also some classes of almost metallic Norden and metallic Riemannian manifolds introduced in the same way (see [2, Prop. 5.1] and [10, Prop. 2.3]).

To use the defining conditions of all classes of $\alpha$-metric manifolds to classify $\alpha$-metallic metric manifolds, we need to relate tensors fields and 1-forms related with $\left(J_{\varphi}, g\right)$ involved in Theorem 2.1 with other ones defined from $(\varphi, g)$. Now we introduce a 1-form on $\alpha$-metallic metric manifolds in analogous way to the codifferential of $\Phi$ of an $\alpha$-metric manifold starting from $(\varphi, g)$ instead of $\left(J_{\varphi}, g\right)$ as follows.

Definition 3.2. Let $(M, \varphi, g)$ be a $2 n$-dimensional $\alpha$-metallic metric manifold. There exist a 1 -form $\delta \varphi$, called the codifferential of $\varphi$, which locally can be defined as follows:

$$
\delta \varphi(X)=\sum_{i, j=1}^{2 n} g^{i j} g\left(\left(\nabla_{X_{i}}^{\mathrm{g}} \varphi\right) X_{j}, X\right) \forall X \in \mathfrak{X}(M),
$$

where $\left(X_{1}, \ldots, X_{2 n}\right)$ is a local basis of $T M$ and the matrix $\left(g^{i j}\right)_{1 \leq i, j \leq 2 n}$ is the inverse matrix of $\left(g\left(X_{i}, X_{j}\right)\right)_{1 \leq i, j \leq 2 n}$

Starting from (1.3), (1.9), (2.16) and (3.31), by straightforward calculations, it is easy to prove the following equalities between tensor fields and 1-forms defined from $(\varphi, g)$ and $\left(J_{\varphi}, g\right)$

$$
\begin{array}{ll}
\left(\nabla_{X}^{\mathrm{g}} J_{\varphi}\right) Y=\frac{2 \alpha}{\sqrt{p^{2}+4 q}}\left(\nabla_{X}^{\mathrm{g}} \varphi\right) Y, & g\left(X, J_{\varphi} Y\right)=\frac{\alpha}{\sqrt{p^{2}+4 q}} g(X, 2 \varphi Y-p Y), \\
\delta \Phi(X)=\frac{2 \alpha}{\sqrt{p^{2}+4 q}} \delta \varphi(X), \quad N_{J_{\varphi}}(X, Y)=\frac{4}{p^{2}+4 q} N_{\varphi}(X, Y), \quad \forall X, Y \in \mathfrak{X}(M) .
\end{array}
$$

The above equalities allow to characterize all classes of $\alpha$-metallic metric manifolds easily according to Definition 3.1. For instance, given $X, Y$ vector fields on $M$, identities 
(3.33) and (3.34) of the next result can be obtained starting from (2.19) and (2.22) respectively bearing in mind (1.3) and (3.32) by means of the next ones

$$
\begin{aligned}
\left(\nabla_{X}^{\mathrm{g}} J_{\varphi}\right) J_{\varphi} X+\left(\nabla_{J_{\varphi} X}^{\mathrm{g}} J_{\varphi}\right) X & =\frac{4}{p^{2}+4 q}\left(\left(\nabla_{X}^{\mathrm{g}} \varphi\right) \varphi X+\left(\nabla_{\varphi X}^{\mathrm{g}} \varphi\right) X-p\left(\nabla_{X}^{\mathrm{g}} \varphi\right) X\right), \\
g\left(X, J_{\varphi} X\right) \delta \Phi(Y)-g(X, X) \delta \Phi\left(J_{\varphi} Y\right) & =\frac{4}{p^{2}+4 q}(g(X, \varphi X) \delta \varphi(Y)-g(X, X) \delta \varphi(\varphi Y)) .
\end{aligned}
$$

Theorem 3.3. Let $(M, \varphi, g)$ be a $2 n$-dimensional $\alpha$-metallic metric manifold. Then one has the following classes of this kind of manifolds:

i) The class $\mathcal{W}_{0}$ or Kähler metallic manifolds characterized by the condition

$$
\nabla^{\mathrm{g}} \varphi=0 .
$$

ii) The class $\mathcal{W}_{1}$ characterized by the condition

$$
\begin{aligned}
g\left(\left(\nabla_{X}^{\mathrm{g}} \varphi\right) Y, Z\right) & =\frac{1}{\left(p^{2}+4 q\right) n} g(X, Y) \delta \varphi\left(\frac{p^{2}(1-\alpha)+4 q}{2} Z+\alpha p \varphi Z\right) \\
& +\frac{1}{\left(p^{2}+4 q\right) n} g(X, Z) \delta \varphi\left(\frac{p^{2}(1-\alpha)+4 q}{2} Y+\alpha p \varphi Y\right) \\
& +\frac{\alpha}{\left(p^{2}+4 q\right) n}(g(X, \varphi Y) \delta \varphi(p Z-2 \varphi Z)+g(X, \varphi Z) \delta \varphi(p Y-2 \varphi Y)),
\end{aligned}
$$

for all vector fields $X, Y, Z$ on $M$.

iii) The class $\mathcal{W}_{2}$ characterized by the conditions

$$
\delta \varphi=0, N_{\varphi}=0 .
$$

$i v)$ The class $\mathcal{W}_{3}$ or quasi-Kähler metallic manifolds characterized by the condition

$$
\left(\nabla_{X}^{\mathrm{g}} \varphi\right) \varphi X+\left(\nabla_{\varphi X}^{\mathrm{g}} \varphi\right) X-p\left(\nabla_{X}^{\mathrm{g}} \varphi\right) X=0, \quad \forall X \in \mathfrak{X}(M) .
$$

v) The class $\mathcal{W}_{1} \oplus \mathcal{W}_{2}$ or integrable metallic manifolds characterized by the condition

$$
N_{\varphi}=0 .
$$

vi) The class $\mathcal{W}_{2} \oplus \mathcal{W}_{3}$ characterized by the condition

$$
\delta \varphi=0 .
$$

vii) The class $\mathcal{W}_{1} \oplus \mathcal{W}_{3}$ characterized by the condition

$$
\begin{aligned}
g\left(\left(\nabla_{X}^{\mathrm{g}} \varphi\right) \varphi X+\left(\nabla_{\varphi X}^{\mathrm{g}} \varphi\right) X-p\left(\nabla_{X}^{\mathrm{g}} \varphi\right) X, Y\right) & =\frac{1}{n} g(X, \varphi X) \delta \varphi(Y) \\
& -\frac{1}{n} g(X, X) \delta \varphi(\varphi Y),
\end{aligned}
$$

for all vector fields $X, Y$ on $M$.

viii) The class $\mathcal{W}$ or the whole class of $\alpha$-metallic metric manifolds.

We introduce the notion of adapted connection on $\alpha$-metallic metric manifolds below.

Definition 3.3. Let $(M, g, \varphi)$ be an $\alpha$-metallic metric manifold and let $\nabla$ be a connection on $M$. One says that $\nabla$ is an adapted connection to $(\varphi, g)$ if it satisfies $\nabla \varphi=0$ and $\nabla g=0$.

The first equality of (3.32) allows us to claim that a connection $\nabla$ on an $\alpha$-metallic metric manifold $(M, \varphi, g)$ is adapted to $(\varphi, g)$ if and only if $\nabla$ is also adapted to $\left(J_{\varphi}, g\right)$.

Using the 1: 1 correspondence between $\alpha$-metric manifolds and $\alpha$-metallic metric manifolds we can attach one distinguished adapted connection to an $\alpha$-metallic metric manifold as follows. 
Definition 3.4. Let $(M, g, \varphi)$ be an $\alpha$-metallic metric manifold. We call the first canonical connection of $(M, \varphi, g)$ to the first canonical connection of its corresponding $\alpha$-metric manifold $\left(M, J_{\varphi}, g\right)$.

Bearing in mind (1.3), (1.7) and (3.32), one obtains that the first canonical connection $\nabla^{0}$ of $(M, \varphi, g)$ is given by

$$
\nabla_{X}^{0} Y=\nabla_{X}^{\mathrm{g}} Y+\frac{\alpha p}{p^{2}+4 q}\left(\nabla_{X}^{\mathrm{g}} \varphi\right) Y-\frac{2 \alpha}{p^{2}+4 q}\left(\nabla_{X}^{\mathrm{g}} \varphi\right) \varphi Y, \quad \forall X, Y \in \mathfrak{X}(M) .
$$

Bearing in mind identity (1.2), it is easy to prove the following equality

$$
\left(\nabla_{X}^{\mathrm{g}} \varphi\right) \varphi Y=p\left(\nabla_{X}^{\mathrm{g}} \varphi\right) Y-\varphi\left(\nabla_{X}^{\mathrm{g}} \varphi\right) Y, \quad \forall X, Y \in \mathfrak{X}(M) .
$$

The above identities leads to the following alternative expression of the first canonical connection

$$
\nabla_{X}^{0} Y=\nabla_{X}^{\mathrm{g}} Y-\frac{\alpha p}{p^{2}+4 q}\left(\nabla_{X}^{\mathrm{g}} \varphi\right) Y+\frac{2 \alpha}{p^{2}+4 q} \varphi\left(\nabla_{X}^{\mathrm{g}} \varphi\right) Y, \quad \forall X, Y \in \mathfrak{X}(M) .
$$

This adapted connection was already introduced on $\alpha$-metallic metric manifolds in another way. The above expression is those that appears in [3, Theor. 3.1].

The first canonical connection allows us to characterize all classes of $\alpha$-metallic metric manifolds carrying the defining conditions of all classes of $\alpha$-metric manifolds collected in Theorem 2.2 using the bijection between both kind of metric manifolds.

Theorem 3.4. Let $(M, \varphi, g)$ be a $2 n$-dimensional $\alpha$-metallic metric manifold. The classes given in Theorem 3.3 can be characterized by means of the first canonical connection as follows:

i) The class $\mathcal{W}_{0}$ or Kähler metallic manifolds characterized by the condition

$$
\mathrm{T}^{0}(X, Y)=0, \forall X, Y \in \mathfrak{X}(M) .
$$

ii) The class $\mathcal{W}_{1}$ characterized by the condition

$$
\begin{aligned}
\mathrm{T}^{0}(X, Y) & =\frac{1}{\left(p^{2}+4 q\right) n} t^{0}\left(\frac{p^{2}(1-\alpha)+4 q}{2} X+\alpha p \varphi X\right) Y \\
& -\frac{1}{\left(p^{2}+4 q\right) n} t^{0}\left(\frac{p^{2}(1-\alpha)+4 q}{2} Y+\alpha p \varphi Y\right) X \\
& +\frac{\alpha}{\left(p^{2}+4 q\right) n}\left(t^{0}(p X-2 \varphi X) \varphi Y-t^{0}(p Y-2 \varphi Y) \varphi X\right),
\end{aligned}
$$

for all vector fields $X, Y$ on $M$.

iii) The class $\mathcal{W}_{2}$ characterized by the conditions

$$
\begin{aligned}
t^{0}(X) & =0, \\
\frac{p^{2}+\left(p^{2}+4 q\right) \alpha}{2} \mathrm{~T}^{0}(X, Y)+2 \mathrm{~T}^{0}(\varphi X, \varphi Y) & =p\left(\mathrm{~T}^{0}(\varphi X, Y)+\mathrm{T}^{0}(X, \varphi Y)\right),
\end{aligned}
$$

for all vector fields $X, Y$ on $M$.

$i v)$ The class $\mathcal{W}_{3}$ or quasi-Kähler metallic manifolds characterized by the condition

$$
\begin{aligned}
\frac{p^{2}+\left(p^{2}+4 q\right) \alpha}{2} g\left(\mathrm{~T}^{0}(X, Y), X\right) & =-2 g\left(\mathrm{~T}^{0}(\varphi X, Y), \varphi X\right) \\
& +p\left(g\left(\mathrm{~T}^{0}(\varphi X, Y), X\right)+g\left(\mathrm{~T}^{0}(X, Y), \varphi X\right)\right),
\end{aligned}
$$

for all vector fields $X, Y, Z \in \mathfrak{X}(M)$. 
v) The class $\mathcal{W}_{1} \oplus \mathcal{W}_{2}$ or integrable metallic manifolds characterized by the condition

$$
\frac{p^{2}+\left(p^{2}+4 q\right) \alpha}{2} \mathrm{~T}^{0}(X, Y)+2 \mathrm{~T}^{0}(\varphi X, \varphi Y)=p\left(\mathrm{~T}^{0}(\varphi X, Y)+\mathrm{T}^{0}(X, \varphi Y)\right),
$$

for all vector fields $X, Y \in \mathfrak{X}(M)$.

vi) The class $\mathcal{W}_{2} \oplus \mathcal{W}_{3}$ characterized by the condition

$$
t^{0}(X)=0, \quad \forall X \in \mathfrak{X}(M) .
$$

vii) The class $\mathcal{W}_{1} \oplus \mathcal{W}_{3}$ characterized by the condition

$$
\begin{aligned}
\frac{p^{2}+\left(p^{2}+4 q\right) \alpha}{2} g\left(\mathrm{~T}^{0}(X, Y), X\right) & =-2 g\left(\mathrm{~T}^{0}(\varphi X, Y), \varphi X\right) \\
& +p\left(g\left(\mathrm{~T}^{0}(\varphi X, Y), X\right)+g\left(\mathrm{~T}^{0}(X, Y), \varphi X\right)\right) \\
& +\frac{1}{n} g(X, X) t^{0}\left(\frac{p^{2}(1-\alpha)-4 q \alpha}{2} Y-p \varphi Y\right) \\
& -\frac{1}{n} g(X, \varphi X) t^{0}(p Y-2 \varphi Y),
\end{aligned}
$$

for all vector fields $X, Y$ on $M$.

viii) The class $\mathcal{W}$ or the whole class of $\alpha$-metallic metric manifolds.

If $(\alpha, p, q)=(1,1,1)$, the above result allows to recover the classification of almost golden Riemannian manifolds with null trace using the first canonical previously obtained in [8, Theor. 4.3]. Obiously, it also allows to recover the classification of $\alpha$-metric manifolds showed in Theorem 2.2 in the case $(p, q)=(0,1)$.

Defining conditions as the above ones can be found in the Mathematical Literature. For instance, in [3, Prop. 3.1], Blaga and Nannicini characterize the class of integrable metallic manifolds by the condition

$$
\mathrm{T}^{0}(\varphi X, Y)+\mathrm{T}^{0}(X, \varphi Y)=p \mathrm{~T}^{0}(X, Y), \quad X, Y \in \mathfrak{X}(M) .
$$

Indeed, according to Definition 3.1, $(M, \varphi, g)$ is an integrable manifold if and only if $\left(M, J_{\varphi}, g\right)$ is an integrable manifold too, class of manifolds characterized by (2.28) which it is equivalent to the next one

$$
\mathrm{T}^{0}\left(J_{\varphi} X, Y\right)+\mathrm{T}^{0}\left(X, J_{\varphi} Y\right)=0, \quad X, Y \in \mathfrak{X}(M),
$$

then taking into account identity (1.3) one obtains the below defining condition equivalent to (3.35)

$$
\mathrm{T}^{0}(p X-2 \varphi X, Y)+\mathrm{T}^{0}(X, p Y-2 \varphi Y)=0, \quad X, Y \in \mathfrak{X}(M) .
$$

Remark 3.1. To classify $\alpha$-metallic metric manifolds as it is indicated in Definition 3.1 allow us to obtain interesting results like the following. In [2, Defin. 3.1], the authors introduced nearly Kähler Norden manifolds $(M, J, g)$ as almost Norden manifolds satisfying $\left(\nabla_{X}^{\mathrm{g}} J\right) Y+\left(\nabla_{Y}^{\mathrm{g}} J\right) X=0$, for all $X, Y$ vector fields on $M$ and they proved that 4-dimensional manifolds in the previous conditions belong to the Kähler class. They also introduced nearly locally metallic manifolds like those almost metallic Norden manifolds such that their corresponding almost Norden manifolds are nearly Kähler Norden manifolds. As direct consequence of [5, Theor. 4], one obtains that nearly Kähler Norden manifolds are always Kähler Norden manifolds. Thus, nearly locally metallic manifolds are always Kähler Norden metallic manifolds.

Acknowledgements. The authors are very grateful to the referees for their careful reading and valuable suggestions. 


\title{
REFERENCES
}

[1] Blaga, A. M.; Crasmareanu, M. Golden-statistical structures. C. R. Acad. Bulgare Sci. 69 (2016), no. 9, 1113-1120.

[2] Blaga, A. M.; Nannicini, A. On curvature tensors of Norden and metallic pseudo-Riemannian manifolds. Complex Manifolds 6 (2019), no. 1, 150-159.

[3] Blaga, A. M.; Nannicini, A. On the geometry of metallic pseudo-Riemannian structures. Riv. Math. Univ. Parma 11 (2020), no. 1, 69-87.

[4] Crasmareanu, M.; Hrețcanu, C. E. Golden differential geometry. Chaos, Solitons Fractals 38 (2008), no. 5, 1229-1238.

[5] Etayo, F.; deFrancisco, A.; Santamaría, R. There are no genuine Kähler-Codazzi manifolds. Int. J. Geom. Methods Mod. Phys. 17 (2020), no. 3, 2050044, 12 pp.

[6] Etayo, F.; deFrancisco, A.; Santamaría, R. Classification of almost Norden golden manifolds. Bull. Malays. Math. Sci. Soc. 43 (2020), no. 6, 3941-3961.

[7] Etayo, F.; Santamaría, R. Distinguished connections on $\left(J^{2}= \pm 1\right)$-metric manifolds. Arch. Math. (Brno) 52 (2016), no. 3, 159-203.

[8] Etayo, F.; Santamaría, R. Classification of almost Golden Riemannian manifolds with null trace. Mediterr. J. Math. 17 (2020), no. 3, Paper No. 90, 19 pp.

[9] Ganchev, G. T.; Borisov, A. V. Note on the almost complex manifolds with a Norden metric. C. R. Acad. Bulgare Sci. 39 (1986), no. 5, 31-34.

[10] Gezer, A.; Karaman, C. On metallic Riemannian structures. Turkish J. Math. 39 (2015), no. 6, $954-962$.

[11] Hreţcanu, C. E.; Crasmareanu, M. Metallic structures on Riemannian manifolds. Rev. Un. Mat. Argentina 54 (2013), no. 2, 15-27.

[12] Staikova, M.; Gribachev, K. I. Canonical connections and their conformal invariants on Riemannian almost product manifolds. Serdica Math. J. 18 (1992), no. 3-4, 150-161.

[13] Turanli, S.; Gezer, A.; Cakicioglu, H. Metallic Kähler and nearly metallic Kähler manifolds. Int. J. Geom. Methods Mod. Phys. 18 (2021), no. 9, Paper No. 2150146, 20 pp.

\author{
Departamento de MATEMÁticas \\ UNIVERSIDAD DE CANTABRIA \\ ESTADÍSTICA Y COMPUTACIÓN \\ FACULTAD DE CIENCIAS \\ AVDA. DE LOS CASTROS, S/N, 39071 SANTANDER, SPAin \\ Email address: fernando. etayo@unican.es \\ Departamento de MATEMÁticas \\ UNIVERSIDAD DE LEÓN \\ ESCUELA DE INGENIERÍAS INDUSTRIAL, INFORMÁTICA Y AEROESPACIAL \\ CAMPUS DE VEGAZANA, 24071 LEÓN, SPAIN \\ Email address: afrai@unileon.es \\ Email address: rsans@unileon.es
}

